\title{
Path Integral Computation of Phonon Anharmonicity
}

\author{
Marco Zoli \\ Istituto Nazionale Fisica della Materia - Universitá di Camerino, \\ 62032 Italy.e-mail: zoli.marco@libero.it
}

(November 15, 2018)

\begin{abstract}
The partition function of an oscillator disturbed by a set of electron particle paths has been computed by a path integral method which permits to evaluate at any temperature the relevant cumulant terms in the series expansion. The time dependent source current peculiar of the semiclassical Su-Schrieffer-Heeger model induces large electron-phonon anharmonicities on the phonon subsystem. As a main signature of anharmonicity the phonon heat capacity shows a peak whose temperature location strongly varies with the strength of the $e-p h$ coupling. High energy oscillators are less sensitive to anharmonic perturbations.
\end{abstract}

71.20.Rv, 31.15.Kb, 63.20.Kr

\section{INTRODUCTION}

There is at present a growing interest in electronphonon non linearities also triggered by the signatures of a large anharmonicity observed in metal diborides [1]. Phonon anharmonicities have a long story in solid state physics closely related to the inelastic neutron scattering theory $[2,3]$. As the anharmonic effects are generally small in crystals up to room temperature, second order perturbation theory suffices to determine the lifetime due to three phonons decay processes while the renormalization of the frequencies, together with the three phonons terms, also requires computation of the four phonons vertex in the first order diagram plus a (dominant) contribution due to the thermal expansion of the crystal [4]. It is known $[5,22,7]$ that most properties of real materials can be well described by replacing the anharmonic phonons with the temperature dependent renormalized harmonic phonons, that is assuming quasi-harmonic vibrational models [8]. Instead, the damping of some anomalous bulk and surface phonons requires explicit computation of the anharmonic interactions $[9,10]$. However, neither first principles calculations of anharmonicities based on density functional perturbation theory [11] nor empirical force constant approaches do separate the bare phononphonon interactions from the non linearities due to the electron-phonon coupling. The latter contribution is generally incorporated in the former by fitting the third and fourth derivatives of the interatomic potential to experimental thermoelastic properties. In systems such as polymers, whose thermal and conducting behavior is shaped by the strength of the e-ph coupling, one would desire to estimate the amount of $e-p h$ anharmonicity which may become relevant in the low temperature region where, instead, the phonon-phonon interactions tend to vanish.

Theoretical investigations on polymers usually depart from the Su-Schrieffer-Heeger (SSH) Hamiltonian [12-14] in which the $e-p h$ coupling is the derivative of the electron hopping integral with respect to the intersite atomic 
displacement. As the electron propagator couples to the oscillator displacement the $\mathrm{SSH}$ model can be attacked by the path integral method [15-17] which allows one to derive the time dependent probability amplitude for a particle in a bath of oscillators [18]. On the other hand, considering the electron particle path as the disturbing source for the phonon subsystem one may evaluate the amount of $e-p h$ anharmonicity by expanding perturbatively the phonon partition function. Analysis of the cumulant terms versus temperature would permit to assess the relevance of the $e-p h$ interactions in the SSH model. This paper addresses precisely this issue focussing on the computation of some equilibrium thermodynamical properties. The path integral method for the model Hamiltonian is outlined in Section II while the results are given in Section III. Section IV contains some final remarks.

\section{HAMILTONIAN MODEL}

In one dimension the SSH Hamiltonian is:

$$
\begin{aligned}
H & =\sum_{r} J_{r, r+1}\left(f_{r}^{\dagger} f_{r+1}+f_{r+1}^{\dagger} f_{r}\right) \\
J_{r, r+1} & =-\frac{1}{2}\left[J+\alpha\left(u_{r}-u_{r+1}\right)\right]
\end{aligned}
$$

where $J$ is the nearest neighbors hopping integral for an undistorted chain, $\alpha$ is the electron-phonon coupling, $u_{r}$ is the displacement of the atomic group on the $r-$ lattice site along the molecular axis, $f_{r}^{\dagger}$ and $f_{r}$ create and destroy electrons (i.e., $\pi$ band electrons in polyacetylene) on the $r$ - group. The non-interacting Hamiltonian is given by a set of independent oscillators. We transform the real space Hamiltonian of (1) into a time dependent Hamiltonian $H\left(\tau, \tau^{\prime}\right)$ by introducing $x(\tau)$ and $y\left(\tau^{\prime}\right)$ as the electron coordinates at the $r$ and $r+1$ lattice sites, respectively. The spacial $e-p h$ correlations contained in (1) are then mapped onto the time axis by changing: $u_{r} \rightarrow u(\tau)$ and $u_{r+1} \rightarrow u\left(\tau^{\prime}\right)$. Accordingly we get:

$$
\begin{aligned}
H\left(\tau, \tau^{\prime}\right) & =J_{\tau, \tau^{\prime}}\left(f^{\dagger}(x(\tau)) f\left(y\left(\tau^{\prime}\right)\right)+f^{\dagger}\left(y\left(\tau^{\prime}\right)\right) f(x(\tau))\right) \\
J_{\tau, \tau^{\prime}} & =-\frac{1}{2}\left[J+\alpha\left(u(\tau)-u\left(\tau^{\prime}\right)\right)\right]
\end{aligned}
$$

Eq.(2) shows the semiclassical nature of the model in which quantum mechanical degrees of freedom interact with the classical variables $u(\tau)$. As the electron hops are not constrained to first neighbors sites along the chain $H\left(\tau, \tau^{\prime}\right)$ is more general than the real space SSH Hamiltonian in (1). Setting $\tau^{\prime}=0, u(0) \equiv y(0) \equiv 0$, averaging the electron operators over the ground state we obtain the average energy per lattice site linearly depending on the displacements:

$$
\begin{aligned}
\frac{L H(\tau)>}{N} & =V(x(\tau))+u(\tau) j(\tau) \\
j(\tau) & =-\alpha(G[-x(\tau),-\tau]+G[x(\tau), \tau])
\end{aligned}
$$


where $N=L / a$, with $L$ the chain length and $a$ the lattice constant. $V(x(\tau))$ (proportional to $J$ ) is the effective term accounting for the $\tau$ dependent electronic hopping while $j(\tau)$ is the external source current for the oscillator field, $G[x(\tau), \tau]$ being the electron propagator.

Taking a large number of oscillators $\left(u_{i}(\tau), i=1 . . \bar{N}\right)$ as the bath for the quantum mechanical particle whose coordinate is $x(\tau)$, the general electron path integral is given by:

$$
\begin{aligned}
& <x(\beta) \mid x(0)>=\prod_{i} \int D u_{i}(\tau) \int D x(\tau) \\
& \times \exp \left[-\int_{0}^{\beta} d \tau \sum_{i} \frac{M_{i}}{2}\left(\dot{u}_{i}{ }^{2}(\tau)+\omega_{i}^{2} u_{i}^{2}(\tau)\right)\right] \\
& \times \exp \left[-\int_{0}^{\beta} d \tau\left(\frac{m}{2} \dot{x}^{2}(\tau)+V(x(\tau))-\sum_{i} u_{i}(\tau) j(\tau)\right)\right]
\end{aligned}
$$

$\beta$ is the inverse temperature, $m$ is the electron mass and $\omega_{i}$ are the oscillators frequencies. The oscillator masses are considered as independent of $i, M_{i} \equiv M$ and hereafter we set $M=10^{4} \mathrm{~m}$. After integrating out the oscillators coordinates over the paths $D u_{i}(\tau)$ and imposing a closure condition $(x(\beta)=x(0))$ on the particle paths, we obtain the total partition function in the functional form:

$$
\begin{aligned}
& Z(j(\tau))=Z_{p h} \oint D x(\tau) \exp \left[-\frac{m}{2} \dot{x}^{2}(\tau)-V(x(\tau))-A(j(\tau))\right] \\
& Z_{p h}=\prod_{i=1}^{\bar{N}} \frac{1}{2 \sinh \left(\hbar \omega_{i} \beta / 2\right)} \\
& A(j(\tau))=-\frac{\hbar^{2}}{4 M} \sum_{i=1}^{\bar{N}} \frac{1}{\hbar \omega_{i} \sinh \left(\hbar \omega_{i} \beta / 2\right)} \\
& \times \int_{0}^{\beta} d \tau j(\tau) \int_{0}^{\beta} d \tau^{\prime \prime} \cosh \left(\omega_{i}\left(\left|\tau-\tau^{\prime \prime}\right|-\beta / 2\right)\right) j\left(\tau^{\prime \prime}\right)
\end{aligned}
$$

The nonequilibrium quantum statistics of the system can be derived via (4) through the closed-time path formalism [19] which permits to evaluate dissipative properties due to the phonon bath friction [20]. The thermodynamical properties of the system follow from (5).

\section{PHONON ANHARMONICITY}

The electron particle path interacts with each of the $\bar{N}$ oscillators through the coupling $\alpha$ (assumed independent of $i$ ) of the SSH Hamiltonian. Then, the $x(\tau)-$ perturbed phonon partition function can be expanded in anharmonic series as: 


$$
\begin{aligned}
Z_{p h}[x(\tau)] \simeq & Z_{p h}\left(1+\sum_{l=1}^{k}(-1)^{l}<C^{l}>_{x}\right) \\
<C^{k}>_{x} & =Z_{p h}^{-1} \prod_{i} \oint D u_{i}(\tau) \frac{1}{k !} \prod_{l=1}^{k}\left[\int_{0}^{\beta} d \tau_{l} u_{i}\left(\tau_{l}\right) j\left(\tau_{l}\right)\right]^{l} \\
& \times \exp \left[-\int_{0}^{\beta} d \tau \sum_{i} \frac{M_{i}}{2}\left(\dot{u}_{i}{ }^{2}(\tau)+\omega_{i}^{2} u_{i}^{2}(\tau)\right)\right]
\end{aligned}
$$

The total phonon partition function $Z_{p h}^{T}$ is obtained by integrating (6) over a set of perturbing electron paths. Since the oscillators are decoupled (and anharmonic effects mediated by the electron particle path are here neglected) we can study the behavior of the cumulant terms $<C^{k}>_{x}$ by selecting a single oscillator having energy $\omega$ and displacement $u(\tau)$.

As a first step let's replace the source current of the SSH model by a linear (in the electron path) current $j_{x}(\tau)=-\alpha x(\tau)$. This allows us to derive analytical expression for the cumulants as shown, for the lowest order, in the Appendix. Let's approximate the electron path by its $\tau$ averaged value: $\langle x(\tau)\rangle \equiv \frac{1}{\beta} \int_{0}^{\beta} d \tau x(\tau)=x_{0} / a$. and expand the oscillator path in $N_{F}$ Fourier components:

$$
\begin{aligned}
& u(\tau)=u_{o}+\sum_{n=1}^{N_{F}} 2\left(\Re u_{n} \cos \left(\omega_{n} \tau\right)-\Im x_{n} \sin \left(\omega_{n} \tau\right)\right) \\
& \omega_{n}=2 \pi n / \beta
\end{aligned}
$$

Taking the measure of integration

$$
\begin{aligned}
\oint D u(\tau) & \equiv\left(\frac{1}{2}\right)^{2 N_{F}} \frac{\left(2 \pi \cdot 2 N_{F} \pi\right)^{2}}{\sqrt{2} \lambda_{M}^{\left(2 N_{F}+1\right)}} \int_{-\infty}^{\infty} d u_{o} \\
& \times \prod_{n=1}^{N_{F}} \int_{-\infty}^{\infty} d \Re u_{n} \int_{-\infty}^{\infty} d \Im u_{n}
\end{aligned}
$$

with $\lambda_{M}=\sqrt{\pi \hbar^{2} \beta / M}$, we obtain for the $k-t h \mathrm{cu}-$ mulant the expression

$<C^{k}>_{N_{F}}=Z_{p h}^{-1} \frac{\left(\alpha_{R} \beta \lambda_{M}\right)^{k}(k-1) ! !}{k ! \pi^{k / 2}(\omega \beta)^{k+1}} \prod_{n=1}^{N_{F}} \frac{(2 n \pi)^{2}}{(2 n \pi)^{2}+(\omega \beta)^{2}}$

$\alpha_{R}=\alpha x_{0} / a$

Let's set $x_{0} / a=0.1$ in the following calculations thus reducing the effective coupling $\alpha_{R}$ by one order of magnitude with respect to the bare value. The trend shown by the results hereafter presented does not depend however on this choice since $x_{0} / a$ and $\alpha$ can be varied independently. As the cumulants should be stable against the number of Fourier components in the oscillator path expansion, using (9) we set the minimum $N_{F}$ through the condition $2 N_{F} \pi \gg \omega \beta$. Noticing that odd $-k$ terms 
vanish the thermodynamics of the anharmonic oscillator can be computed by the cumulant corrections to the harmonic phonon free energy:

$$
F^{(k)}=-\frac{1}{\beta} \ln \left[1+\sum_{l=1}^{k}<C_{N_{F}}^{2 l}>\right]
$$

To proceed I need a criterion to find the temperature dependent cutoff $k^{*}$ in the cumulant series. In the low $T$ limit the third law of thermodynamics may offer the suitable constraint to determine $k^{*}$. Then, given $\alpha$ and $\omega$, the program searches for the cumulant order such that the heat capacity and the entropy tend to zero in the zero temperature limit. At any finite temperature $\mathrm{T}$, the constant volume heat capacity is computed as

$$
\begin{aligned}
C_{V}^{(k)}(T)= & -\left[F^{(k)}(T+2 \Delta)-2 F^{(k)}(T+\Delta)+F^{(k)}(T)\right] \\
& \times\left(\frac{1}{\Delta}+\frac{T}{\Delta^{2}}\right)
\end{aligned}
$$

$\Delta$ being the incremental step and $k^{*}$ is determined as the minimum value for which the heat capacity converges with an accuracy of $10^{-4}$. Figs.1(a) and 1(b) show phonon heat capacity and free energy respectively in the case of a low energy oscillator for an intermediate value of $e$-ph coupling. Harmonic functions, anharmonic functions with second order cumulant and anharmonic functions with $k^{*}$ corrections are reported on in each figure. The second order cumulant is clearly inadequate to account for the low temperature trend yielding a negative phonon heat capacity below $\sim 40 K$ while at high $T$ the second order cumulant contribution tends to vanish. Instead, the inclusion of $k^{*}$ terms in eqs.(10) and (11) leads to the correct zero temperature limit although there is no visible anharmonic effect on the phonon heat capacity throughout the whole temperature range being $C_{V}^{\left(k^{*}\right)}$ perfectly superimposed to the harmonic $C_{V}^{h}$. Note in Fig.1(b) that the $k^{*}$ corrections simply shift downwards the free energy without changing its slope versus temperature. By increasing $\alpha_{R}$, the low $\mathrm{T}$ range with wrong (negative) $C_{V}^{(2)}$ broadens (see Fig.2(a)) whereas the $k^{*}$ contributions permit to fulfill the zero temperature constraint and substantially lower the phonon free energy (see Fig.2(b)). Thus, for the particular choice of constant (in $\tau$ ) source current we find that the $e$-ph anharmonicity renormalizes the phonon partition function although no change occurs in the thermodynamical behavior of the free energy derivatives. Anharmonicity is essential to stabilize the system but it leaves no trace in the heat capacity. Incidentally we note that a similar role for the anharmonic interactions has been proposed to explain the low frequency behavior of glasses [21]. Figure 3 (a) displays the $k^{*}$ temperature dependence for three choices of $e$-ph coupling in the case of a low energy oscillator: while, at high $T$, the number of required cumulants ranges between six and ten according to the coupling, $k^{*}$ strongly grows at low temperatures getting the value 
100 at $T=1 K$ for $\alpha_{R}=60 \mathrm{meV}^{\circ} \AA^{-1}$. The $k^{*}$ versus $\alpha_{R}$ behavior is pointed out in Fig.3(b) for three selected temperatures. Figure 3(c) shows that by decreasing the oscillator energy an increasing number of cumulants has to be taken into account to make the thermodynamical functions convergent: this is consistent with the expectations that high energy oscillators are less sensitive to $e$-ph induced anharmonicity.

Next we turn to the computation of the equilibrium thermodynamics of the phonon subsystem in our semiclassical SSH model hence, using in (6) the external source current given in (3). As the electron propagator depends on the bare hopping integral we set $J=100 \mathrm{meV}$ thus assuming a narrow band electron system. Any electron path yields in principle a different cumulant contribution as pointed out in the Appendix. Numerical investigation shows however that convergent $k$ - order cumulants are achieved by: i) taking $M_{F}=2$ Fourier components in the electron path expansion, ii) setting for the coefficients $\left\{x_{o}, c_{m}\right\}$ a maximum amplitude of order 0.1 (in units of the lattice constant) and iii) summing over $\sim 5^{2 M_{F}+1}$ electron paths.

As in the previous case, we truncate the cumulant series in (6) by invoking the third law of thermodynamics to determine the cutoff $k^{*}$ in the low temperature limit and by searching numerical convergence on the first and second free energy derivatives at any finite temperature. $k^{*}$ does not depend on the specific electron path coefficients then, $<C^{k^{*}}>=\sum_{\left(x_{o}, c_{m}\right)}<C^{k^{*}}>_{\left(x_{o}, c_{m}\right)}$. Again, we can start our analysis from (10) after checking that odd $k$ cumulants yield vanishing contributions. Now however the picture of the anharmonic effects changes drastically. The $e$ - $p h$ coupling strongly modifies the shape of the heat capacity and free energy plots with respect to the harmonic result as it is seen in Figs.4(a) and 4(b) respectively. Above a threshold value $\alpha \sim 10 \mathrm{meV}^{\circ} \AA^{-1}$ (depending on the energy oscillator here set at $\omega=20 \mathrm{meV}$ ) the heat capacity versus temperature curves show a peak whose location along the $T$ axis is reported on in Fig.4(d) as a function of $\alpha$. By enhancing $\alpha$ the height of the peak grows and the bulk of the anharmonic effects on the heat capacity is shifted towards lower $T$. At $\alpha \sim 60 \mathrm{meV} \AA^{-1}$ the crossover temperature is around $100 \mathrm{~K}$. At high $T$ the anharmonic corrections renormalize downwards the free energy but their effect on the heat capacity tends to decrease signalling that $e-p h$ nonlinearities are rather to be seen in the intermediate to low temperature range.

Also the $k^{*}$ versus $T$ behavior is much different from the previous case (discussed in Fig.3(a)): it is seen in Fig.4(c) that a few cumulant terms suffice at low temperatures even at large $e$-ph couplings while $k^{*}$ grows by increasing $T$ for any $\alpha$. Then, $k^{*}(T)$ does not necessarily provide a measure of the degree of $e$-ph anharmonicity as revealed by the heat capacity behavior. At room temperature we find $k^{*}=14$ as the maximum value for the range of $\alpha$ 's here considered with a low energy oscillator. However, by taking $\omega=100 \mathrm{meV}, k^{*}$ drops (as shown in 
Fig.4(c)) attaining the value 6 at around room temperature for $\alpha=60 \mathrm{meV} \stackrel{\circ}{A^{-1}}$. This would suggest that also in the $\mathrm{SSH}$ model high energy phonons are expected to be less anharmonic. This idea is confirmed by looking at Fig.4(d) where the crossover temperature in the oscillator heat capacity curves is reported on, at fixed $\alpha$, versus $\omega$. The heat capacity peak shows up at $\omega \leq 50 \mathrm{meV}$ and its temperature location is shifted downwards by decreasing the phonon energy whereas, at larger energies, the heat capacity has a positive derivative in the whole range of temperatures. Finally we note that recent numerical studies of a classical one dimensional anharmonic model undergoing a Peierls instability [22] also find a specific heat peak as a signature of anharmonicity.

\section{CONCLUSIONS}

We have studied the equilibrium thermodynamics of an electron-phonon system looking in particular at the anharmonic effects produced by the electronic subsystem on the phonon oscillators. The path integral formalism permits to analyse the $e$ - $p h$ non linearities as a function of the source current (peculiar of the Hamiltonian model) which disturbs the harmonic oscillator. The phonon partition function has been expanded in $k$ - order cumulant series and, at any temperature, we have determined the cutoff $k^{*}$ that makes first and second free energy derivatives convergent. In the zero temperature limit the constraint imposed by the third law of thermodynamics has been fulfilled. Rather than being a unique measure of $e$-ph anharmonicity $k^{*}(T)$ turns out to be a model dependent function whose values may vary considerably according to the physical quantities one decides to sample. As a general trend we find however that, at fixed $T$ and $e$-ph coupling, higher energy oscillators are more stable and require a lower $k^{*}$ for the computation of their thermodynamical properties.

We have first considered the case of a source current proportional to the time averaged electron path and analytically derived the cumulant expansion for the phonon partition function. This current induces a slope preserving shift in the phonon free energy versus temperature hence, the heat capacity does not show any correction with respect to the harmonic result in spite of the high number of anharmonic terms which appear in the cumulant series.

Turning to the source current peculiar of the time dependent, one dimensional, semiclassical Su-SchriefferHeeger model we find a striking evidence of $e$-ph anharmonicity in the constant volume heat capacity of a single oscillator. As a main feature the heat capacity exhibits a peak whose height and location on the $T$ axis varies with the strength of the $e$-ph coupling and the energy of the oscillator: while strong couplings shift the main body of the anharmonic effects towards low $T$, high energy phonons prove to be less affected by $e$-ph corrections. 
FIG. 1. (a) Phonon heat capacity and (b) Phonon free energy calculated in i) the harmonic model, ii) anharmonic model with second order cumulant, iii) anharmonic model with $k^{*}$ cumulants (see text). $\alpha_{R}$ is the effective $e$-ph coupling in units $m e V \AA^{-1}$ and $\omega$ is the phonon energy. 
FIG. 2. As in Fig.1 but with larger e-ph coupling.

FIG. 3. (a) Number of cumulants required to obtain a convergent phonon heat capacity at any temperature for different choices of $e-p h$ couplings. (b) Number of cumulants yielding a convergent phonon heat capacity at any $e$-ph coupling for three selected temperatures. (c) Number of cumulants yielding a convergent phonon heat capacity at any oscillator energy for three selected temperatures. 
FIG. 4. Anharmonic (a) phonon heat capacity and (b) free energy versus temperature for eight values of $e$-ph coupling. The harmonic plots are also reported on. A low energy oscillator is assumed. (c) Temperature dependence of the cutoff $k^{*}$ for: i) the weakest and strongest $e$ - $p h$ couplings given in (a) and (b) with $\omega=20 \mathrm{meV}$, ii) the strongest coupling case with $\omega=100 \mathrm{meV}$. (d) Temperatures at which the heat capacity gets the maximum value (see (a)) versus $e$ - $p h$ coupling at fixed $\omega$ (bottom axes) and versus $\omega$ at fixed coupling (top axes).

\section{APPENDIX}

Let's assume a source current $j_{x}(\tau)=-\alpha x(\tau)$ as the disturbing term for the oscillator field, $\alpha$ being the $e$ ph coupling and $x(\tau)$ being the electron path. Then the $k$-th order cumulant term contributing to the $x(\tau)-$ perturbed phonon partition function is given by

$$
\begin{aligned}
<C^{k}>_{x}= & Z_{p h}^{-1} \frac{\alpha^{k}}{k !} \oint D u(\tau) \prod_{l=1}^{k}\left[\int_{0}^{\beta} d \tau_{l} u\left(\tau_{l}\right) x\left(\tau_{l}\right)\right]^{l} . \\
& \exp \left[-\int_{0}^{\beta} d \tau \sum_{i} \frac{M_{i}}{2}\left(\dot{u}^{2}(\tau)+\omega^{2} u^{2}(\tau)\right)\right]
\end{aligned}
$$

We use the oscillator path and the functional measure of integration given in the eqs. (7) and (8) of the text respectively. Then, the electron path expansion in Fourier components

$$
x(\tau)=x_{o}+\sum_{m=1}^{M_{F}}\left(c_{m} \cos \left(\omega_{m} \tau\right)+d_{m} \sin \left(\omega_{m} \tau\right)\right)
$$




$$
\begin{aligned}
& \omega_{m}=2 \pi m / \beta \\
& c_{m}=2 \Re x_{m} \\
& d_{m}=-2 \Im x_{m}
\end{aligned}
$$

will be truncated at $M_{F}=N_{F}$. Without any loss of generality we set $d_{m}=0$ and derive the following expressions for the lowest even order cumulants:

$$
\begin{aligned}
&<C^{2}>_{\left(x_{o}, c_{m}\right)}=Z_{p h}^{-1} \frac{\alpha^{2}}{2} \frac{\left(\beta \lambda_{M}\right)^{2}}{\beta \omega} F\left(N_{F}\right) \cdot\left[\frac{x_{o}^{2}}{\pi(\beta \omega)^{2}}+\right. \\
&\left.\frac{1}{2 \pi} \sum_{m=1}^{N_{F}} \frac{c_{m}^{2}}{(2 \pi m)^{2}+(\beta \omega)^{2}}\right] \\
&<C^{4}>_{\left(x_{o}, c_{m}\right)}=Z_{p h}^{-1} \frac{\alpha^{4}}{4 !} \frac{\left(\beta \lambda_{M}\right)^{4}}{\beta \omega} F\left(N_{F}\right) \cdot\left[\frac{3 x_{o}^{4}}{2 \pi^{2}(\beta \omega)^{4}}+\right. \\
& \frac{3 x_{o}^{2}}{2 \pi^{2}(\beta \omega)^{2}} \sum_{m=1}^{N_{F}} \frac{2 c_{m}^{2}}{(2 \pi m)^{2}+(\beta \omega)^{2}}+ \\
& \frac{3}{8 \pi^{2}} \sum_{m=1, l>m}^{N_{F}} \frac{2 c_{m}^{2}}{(2 \pi m)^{2}+(\beta \omega)^{2}} \cdot \frac{2 c_{l}^{2}}{(2 \pi l)^{2}+(\beta \omega)^{2}}+ \\
&\left.\frac{3}{4 \pi^{2}} \sum_{m=1}^{N_{F}} \frac{c_{m}^{4}}{\left((2 \pi m)^{2}+(\beta \omega)^{2}\right)^{2}}\right] \\
& F\left(N_{F}\right)= \prod_{m=1}^{N_{F}} \frac{(2 \pi m)^{2}}{(2 \pi m)^{2}+(\beta \omega)^{2}}
\end{aligned}
$$

Odd $k$ - cumulants vanish at any order. Setting a maximum amplitude for the coefficients $x_{o}$ and $c_{m}$ and integrating eqs.(14) over a class of electron paths one finds the total cumulant contributions to the partition function.

[1] T.Yildirim, O.Gülseren, J.W.Lynn, C.M.Brown, T.J.Udovic, H.Z.Qing, N.Rogado, K.A.Regan, M.A.Hayward, J.S.Slusky, T.He, M.K.Haas, P.Khalifah, K.Inumaru and R.J.Cava, Phys. Rev. Lett. 87, 037001 (2001).

[2] R.Weinstock, Phys.Rev. 64, 1 (1944).

[3] A.A.Maradudin, A.E.Fein, Phys.Rev. 128, 2589 (1962).

[4] M.Zoli, Phys.Rev.B 41, 7497 (1990).

[5] T.H.K.Barron, in Lattice Dynamics, edited by R.F.Wallis (Pergamon, Oxford, 1965).

[6] J.C.K. Hui, P.B.Allen, J.Phys.C: Solid State Phys. 8, 2923 (1975).

[7] J. K. Freericks, V. Zlatic, M. Jarrell, Phys. Rev. B 61, R838 (2000).

[8] M.Zoli, V.Bortolani, J.Phys.:Condens.Matter 2, 525 (1990).

[9] C.S.Jayanthi, G.Armand, J.R.Manson, Surf.Sci. 154, L247 (1985). 
[10] M.Zoli, Solid State Commun. 80, 173 (1991).

[11] A.Debernardi, F.de Geuser, J.Kulda, M.Cardona, E.E.Haller, cond-mat/0212209

[12] W.P.Su, J.R.Schrieffer, A.J.Heeger, Phys. Rev. Lett. 42, 1698 (1979); A.J. Heeger, S.Kivelson, J.R.Schrieffer, W.P.Su, Rev. Mod. Phys. 60, 781 (1988).

[13] K.Michielsen, H.de Raedt, Z.Phys.B 103, 391 (1997).

[14] N.Miyasaka,Y.Ono, J.Phys.Soc.Jpn. 70, 2968 (2001).

[15] R.P.Feynman, Phys. Rev. 97, 660 (1955).

16] Proceedings of the 6th International Conference on: Path Integrals from peV to Tev - 50 Years after Feynman's paper, World Scientific Publishing (1999).

[17] H.Kleinert, Path Integrals in Quantum Mechanics, Statistics and Polymer Physycs World Scientific Publishing, Singapore (1995).

[18] M.Zoli, Phys. Rev. B 67, 195102 (2003).

[19] J.Schwinger, J.Math.Phys. 2, 407 (1961).

[20] Z.Haba, H.Kleinert, Eur. Phys. J. B 21, 553 (2001).

[21] V.L. Gurevich, D.A. Parshin, H.R. Schober, Phys. Rev. B 67, 094203 (2003).

[22] V.Perebeinos, P.B.Allen, J.Napolitano, Solid State Commun. 118, 215 (2001). 


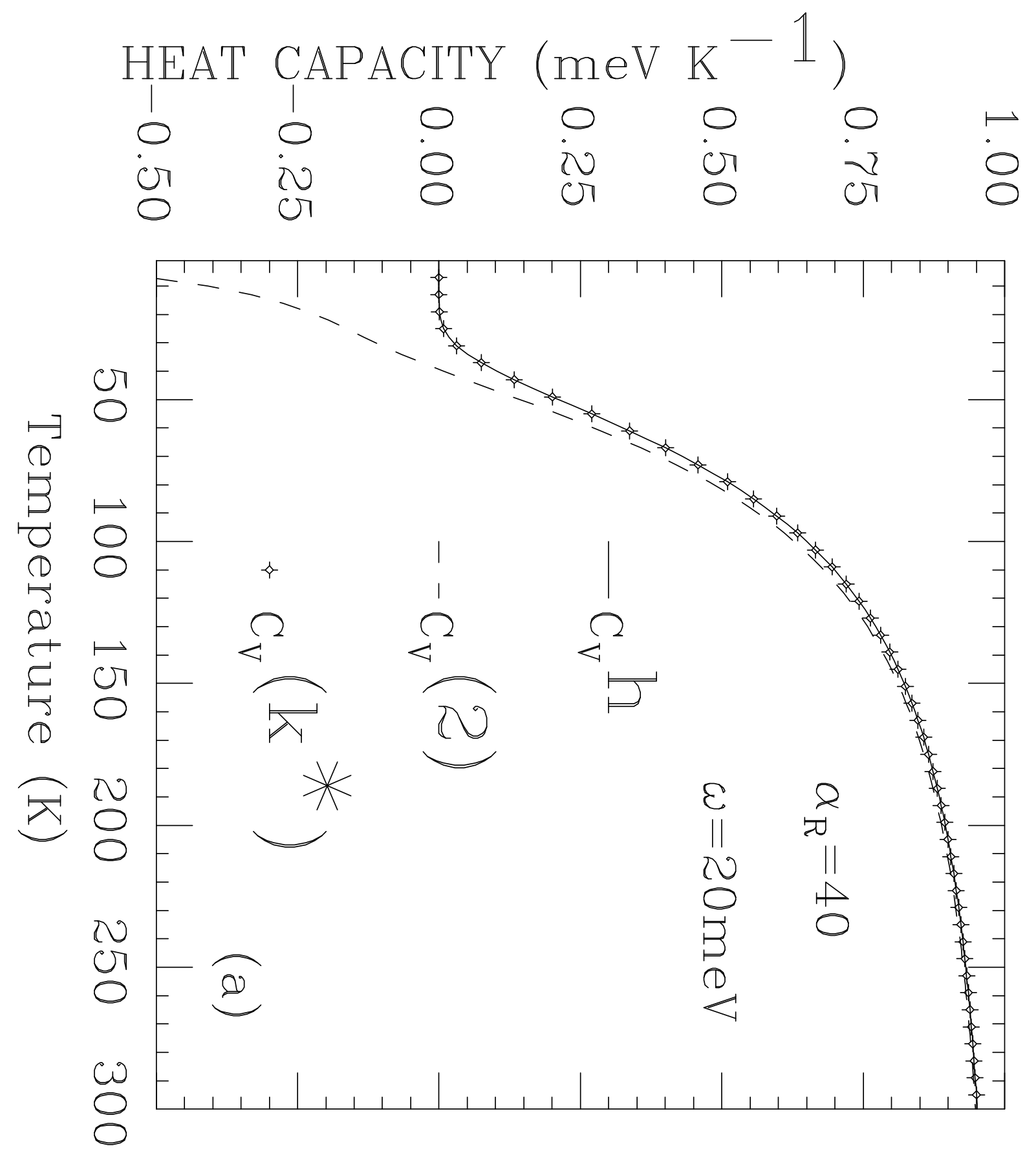


FREE ENERGY (meV)

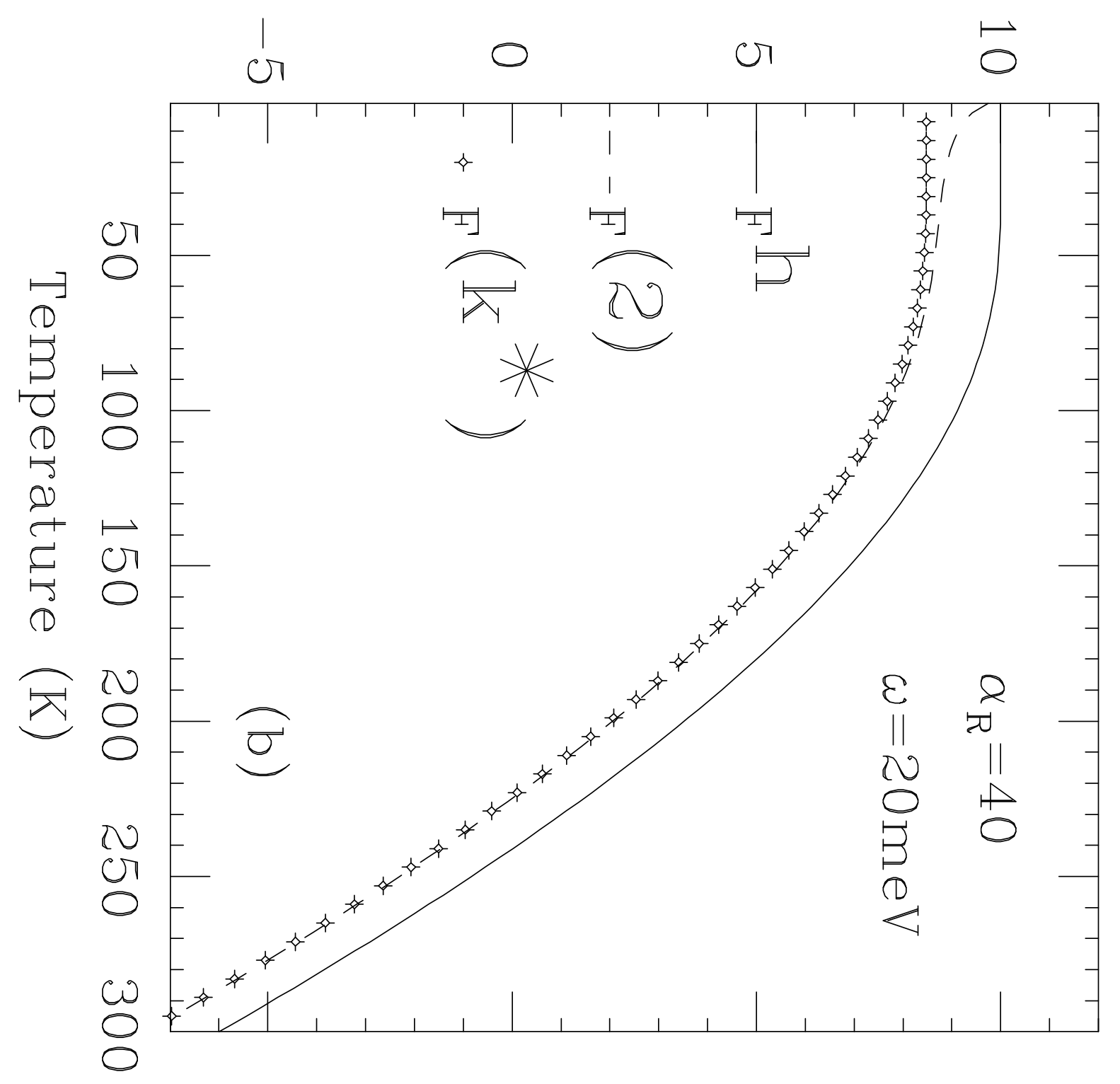




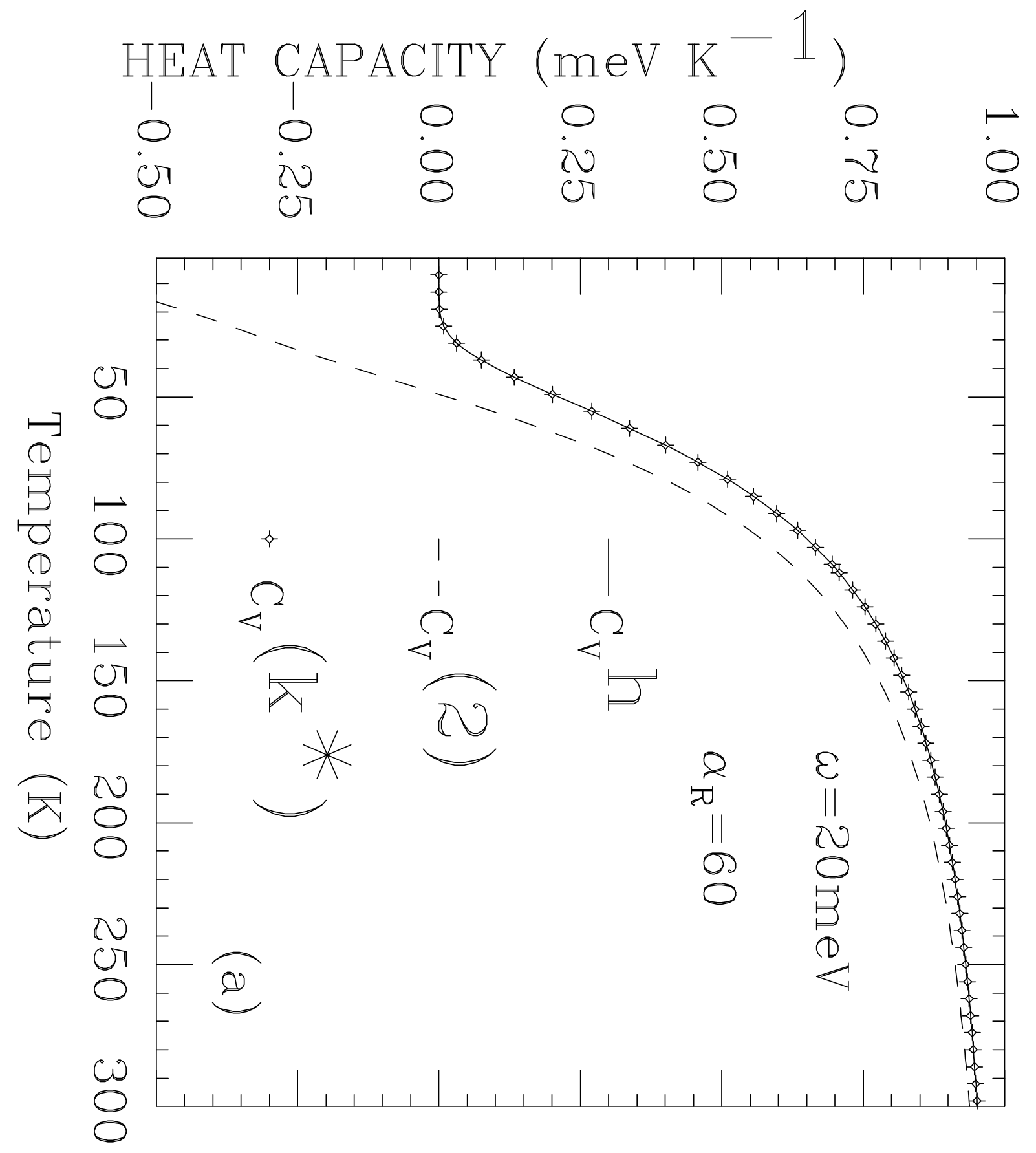


FREE ENERGY (meV)

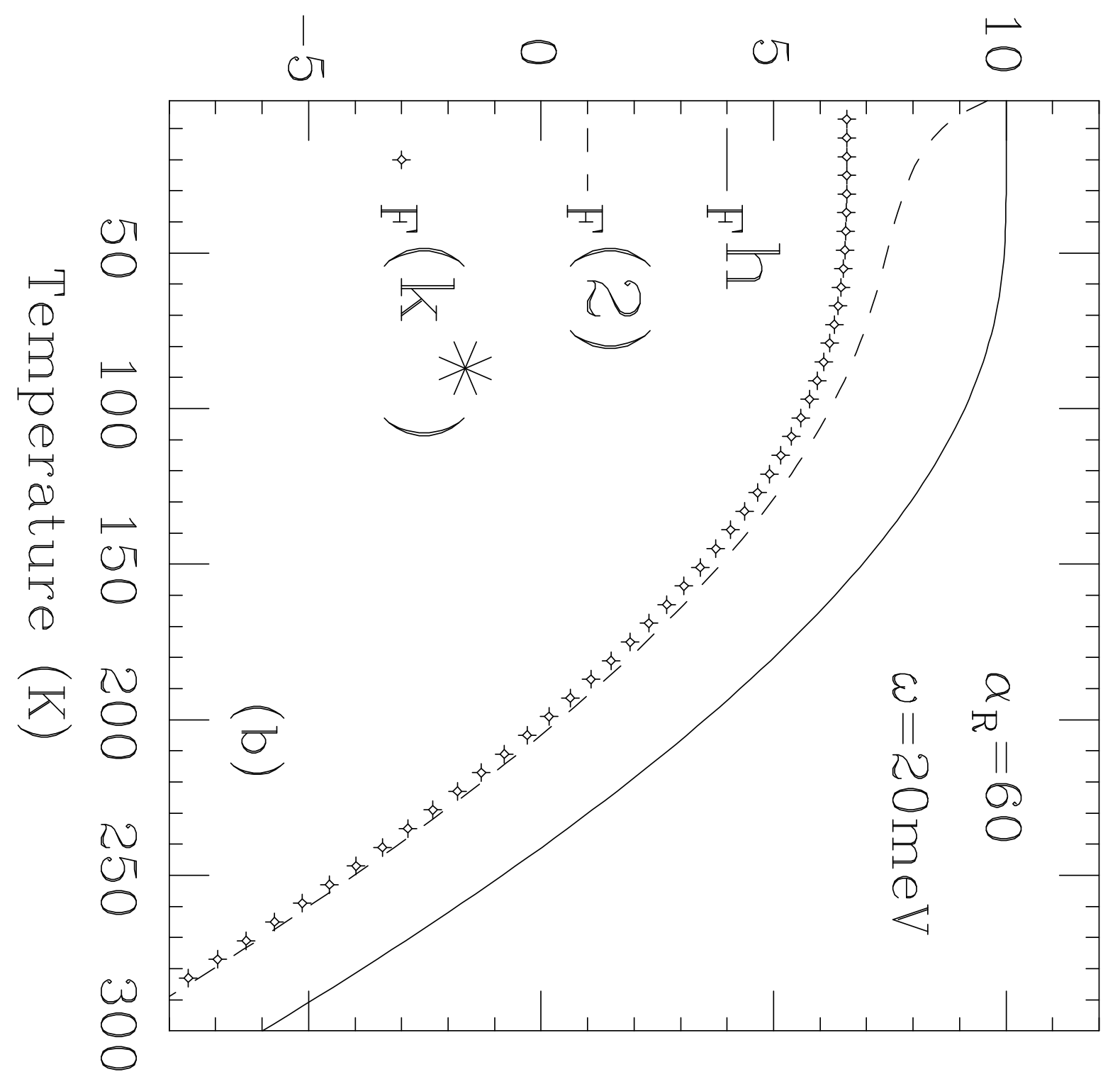




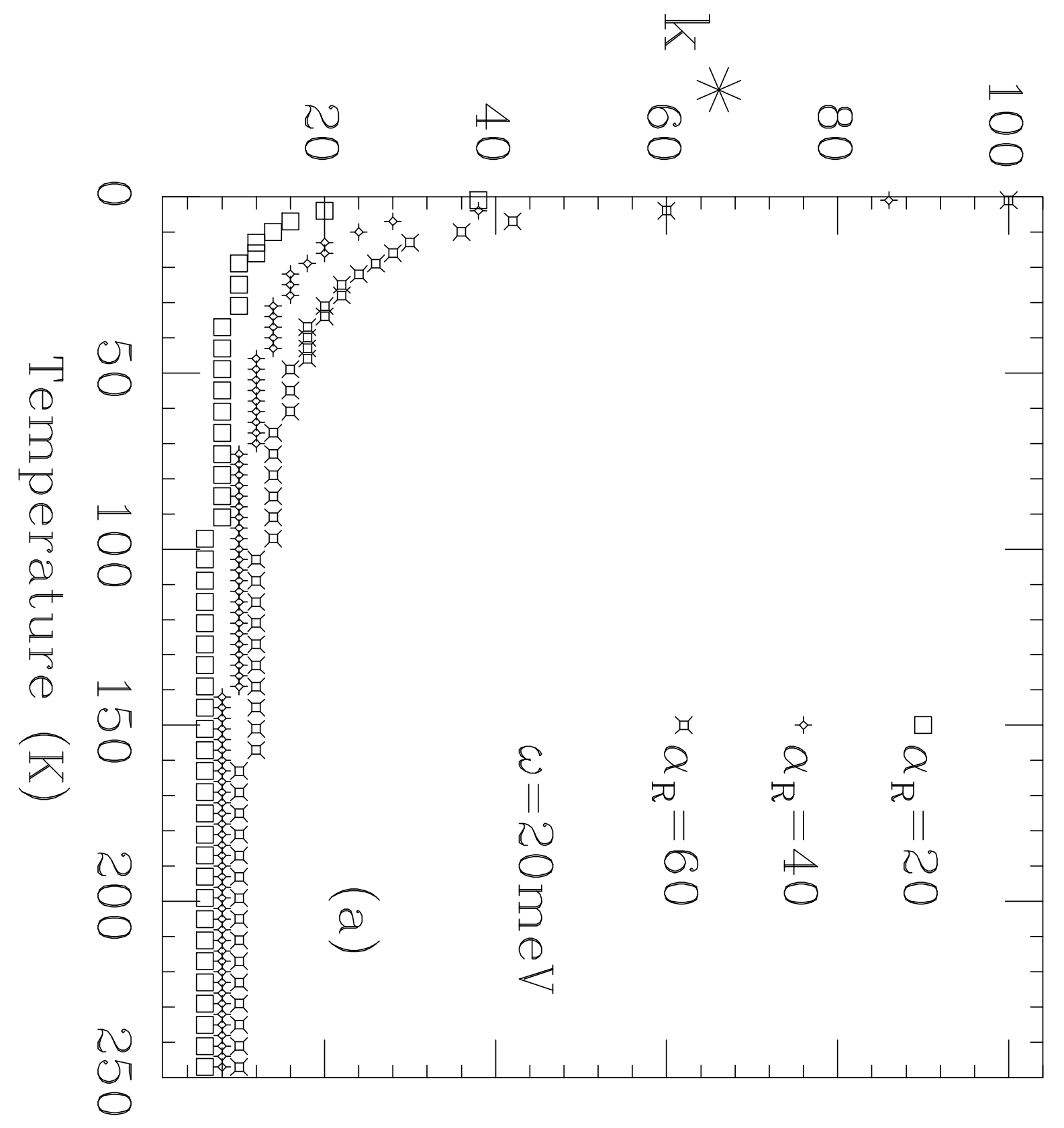




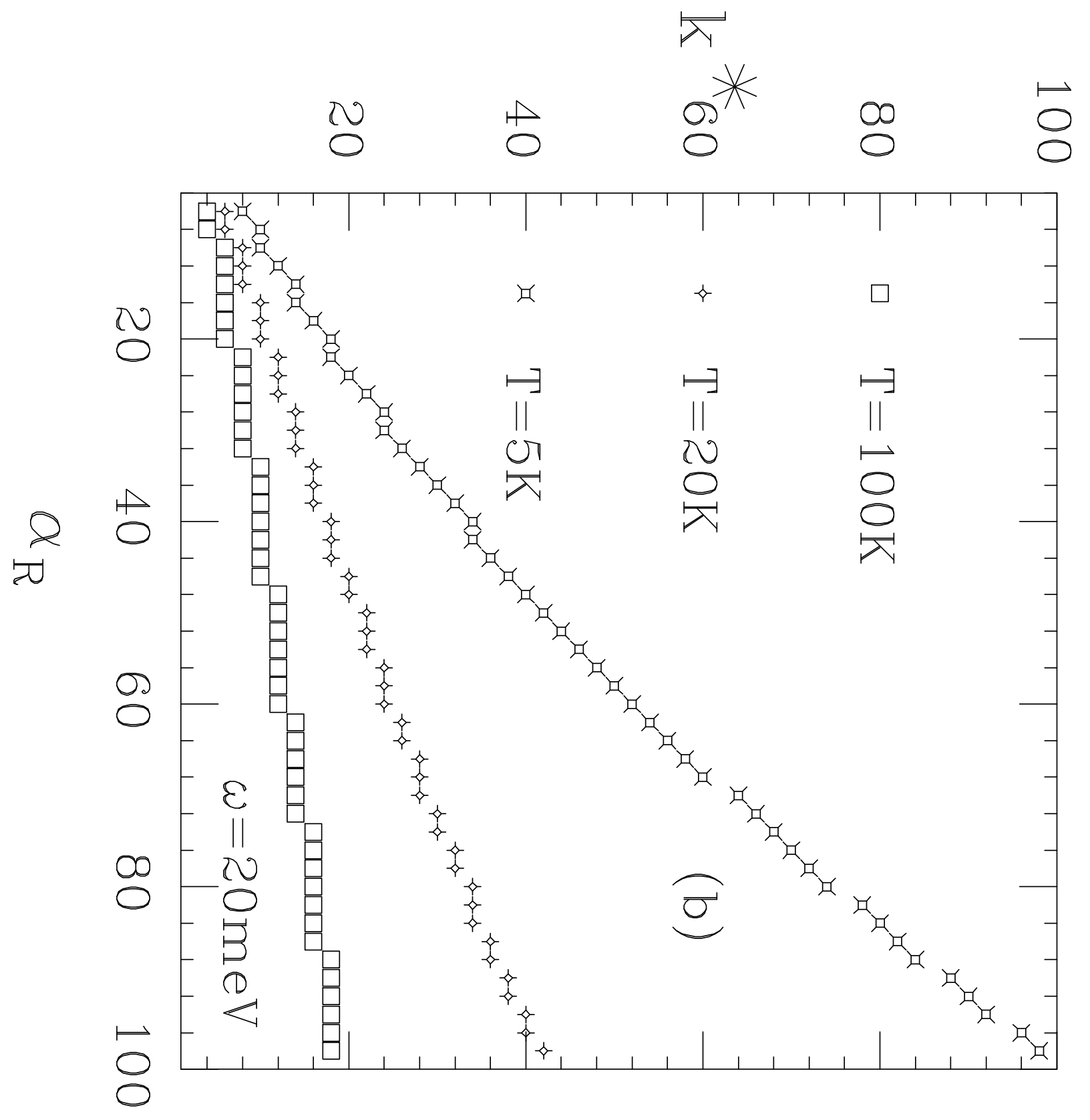




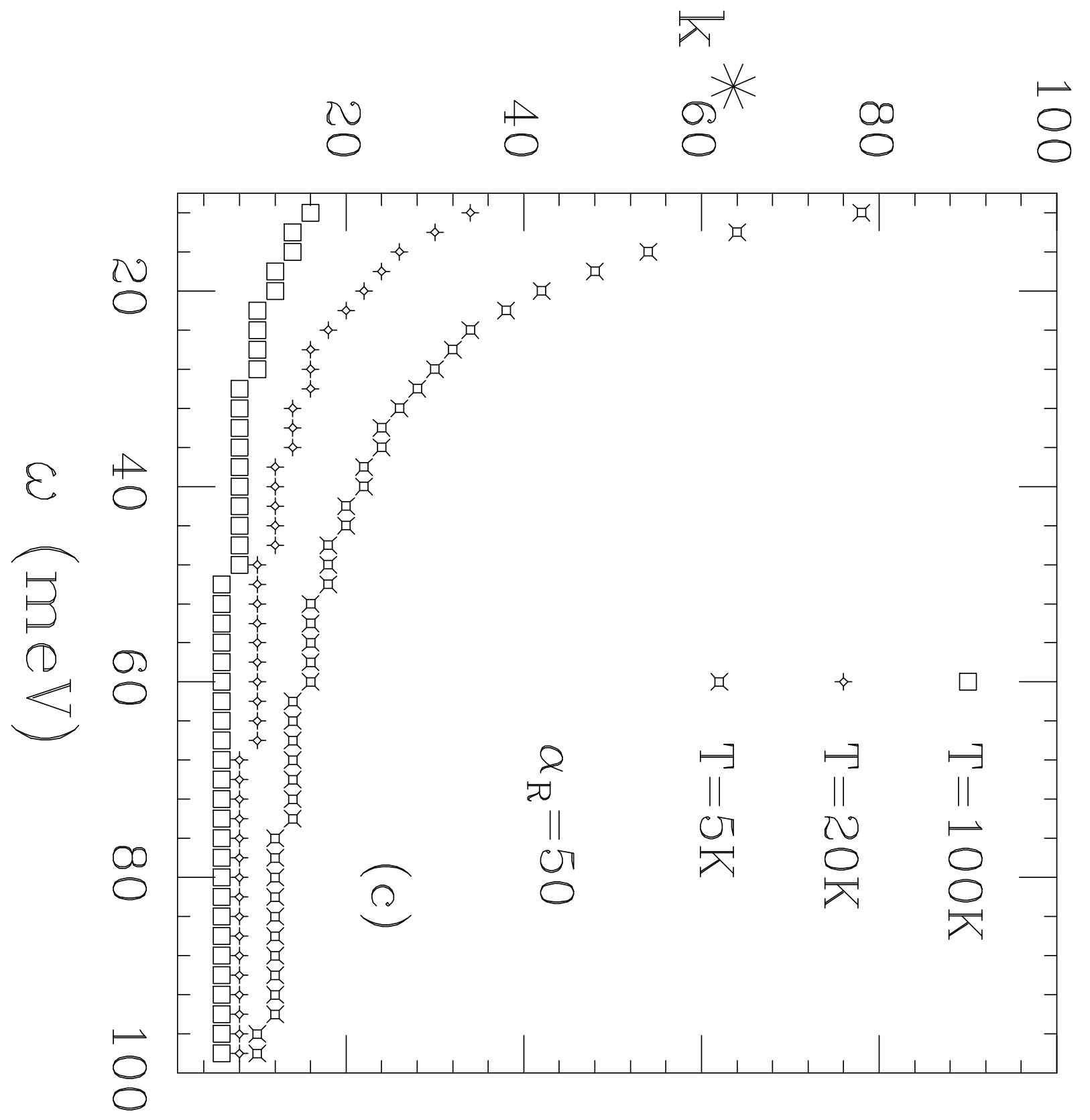




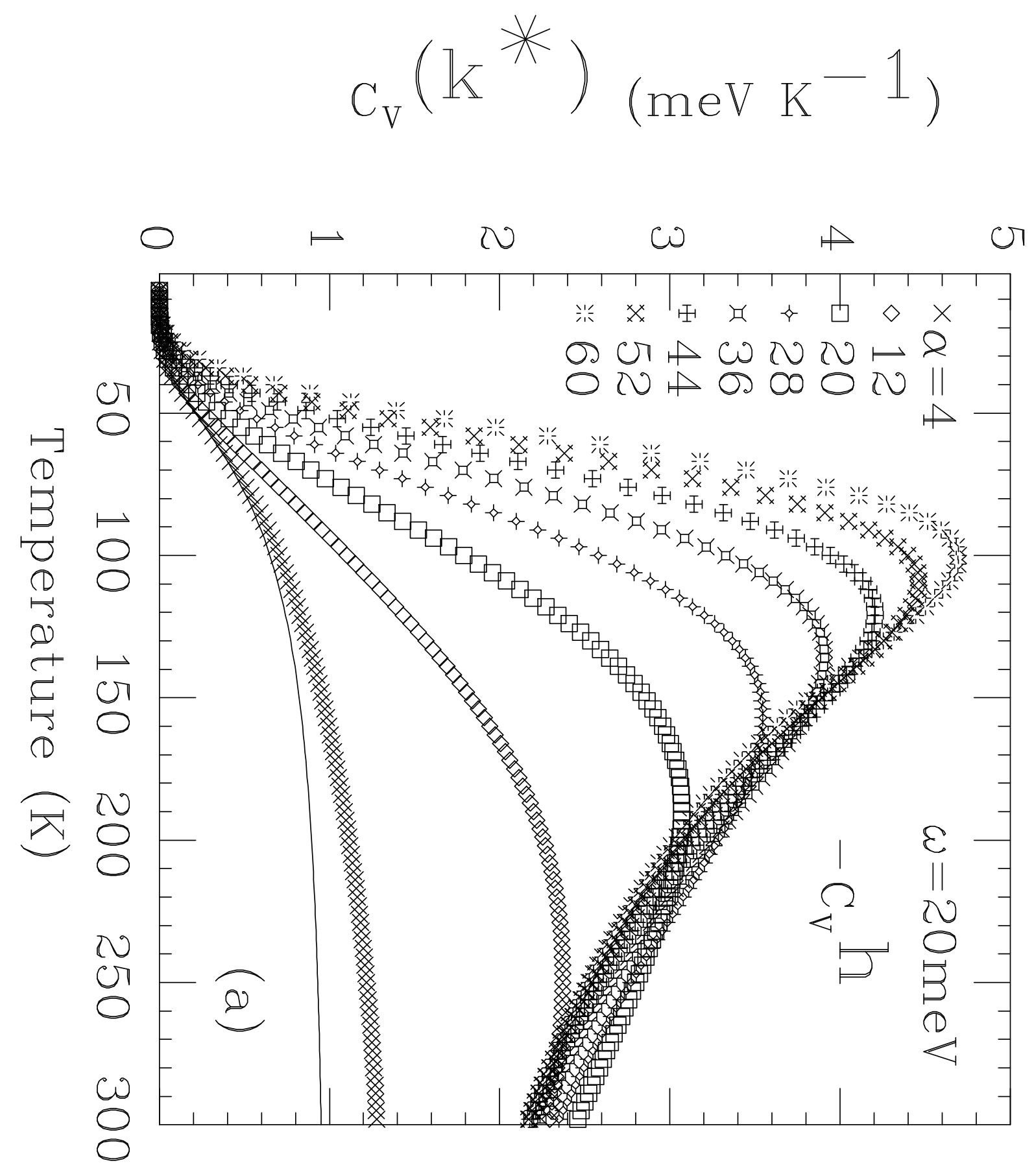




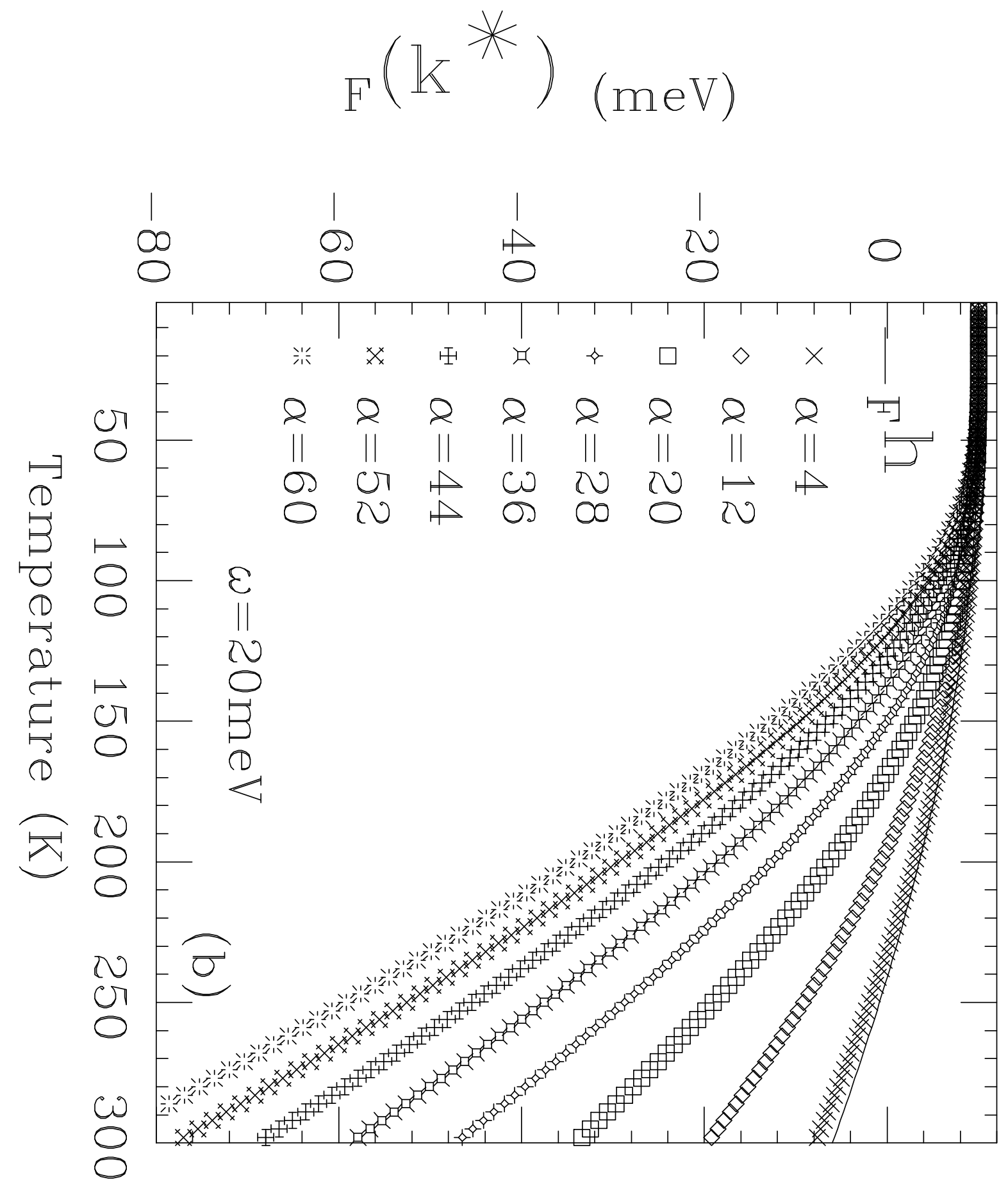




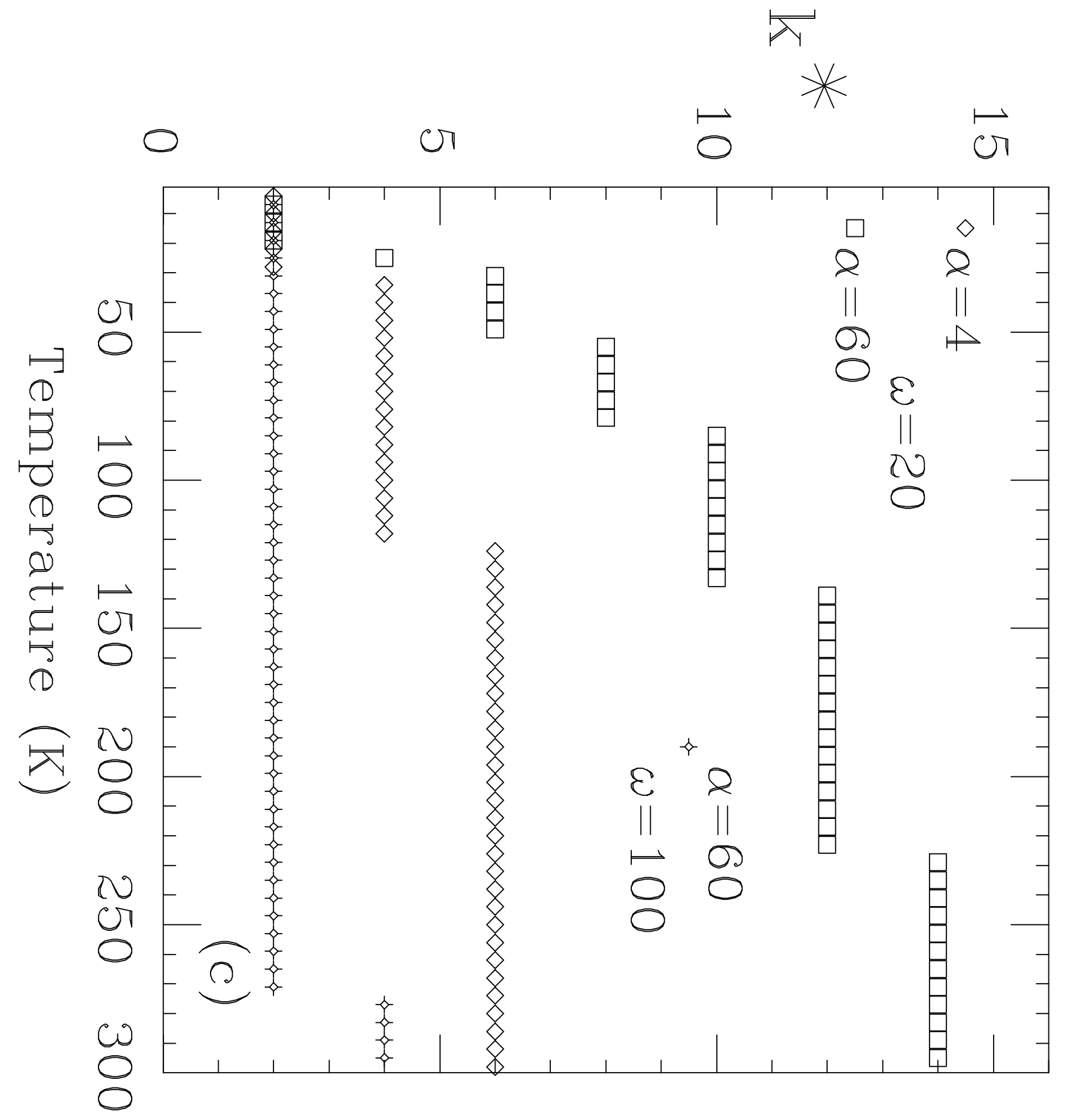

